\title{
From the adiabatic piston to macroscopic motion induced by fluctuations
}

\author{
J. Piasecki \\ Institute of Theoretical Physics, University of Warsaw, Hoża 69, PL-00 681 Warsaw, Poland \\ Ch. Gruber \\ Institut de Physique Théorique, Ecole Polytechnique Fédérale de Lausanne, CH-1015 Lausanne, \\ Switzerland
}

(August 16, 2018)

\begin{abstract}
The controversial problem of the evolution of an isolated system with an internal adiabatic wall is investigated with the use of a simple microscopic model and the Boltzmann equation. In the case of two infinite volume onedimensional ideal fluids separated by a piston whose mass is equal to the mass of the fluid particles we obtain a rigorous explicit stationary non-equilibrium solution of the Boltzmann equation. It is shown that at equal pressures on both sides of the piston, the temperature difference induces a non-zero average velocity, oriented toward the region of higher temperature. It thus turns out that despite the absence of macroscopic forces the asymmetry of fluctuations results in a systematic macroscopic motion. This remarkable effect is analogous to the dynamics of stochastic ratchets, where fluctuations conspire with spatial anisotropy to generate directed motion. However, a different mechanism is involved here. The relevance of the discovered motion to the adiabatic piston problem is discussed.
\end{abstract}

Typeset using REVTEX 


\section{INTRODUCTION}

The recent work of E.Lieb and J.Yngvason [1] on the foundations of thermostatics has given a new interest to an old but still controversial problem of thermodynamics. This is the so called "adiabatic piston problem" described as follows [2] (see also recent references in [3]). The system is composed of a finite volume cylinder containing two fluids separated by an adiabatic movable piston, as shown in Fig.1. A brake maintains initially the piston at rest, and the two fluids are in equilibrium with temperature, pressure and volume given by $\left(T_{1}^{(0)}, p_{1}^{(0)}, V_{1}^{(0)}=A\left(L_{1}+X^{(0)}\right)\right)$, and $\left(T_{2}^{(0)}, p_{2}^{(0)}, V_{2}^{(0)}=A\left(L_{2}-X^{(0)}\right)\right)$, respectively. $A$ denotes here the cross section area of the cylinder, and $X^{(0)}$ the initial position of the piston. At a certain time the brake is released, and the problem is to find the final equilibrium state.

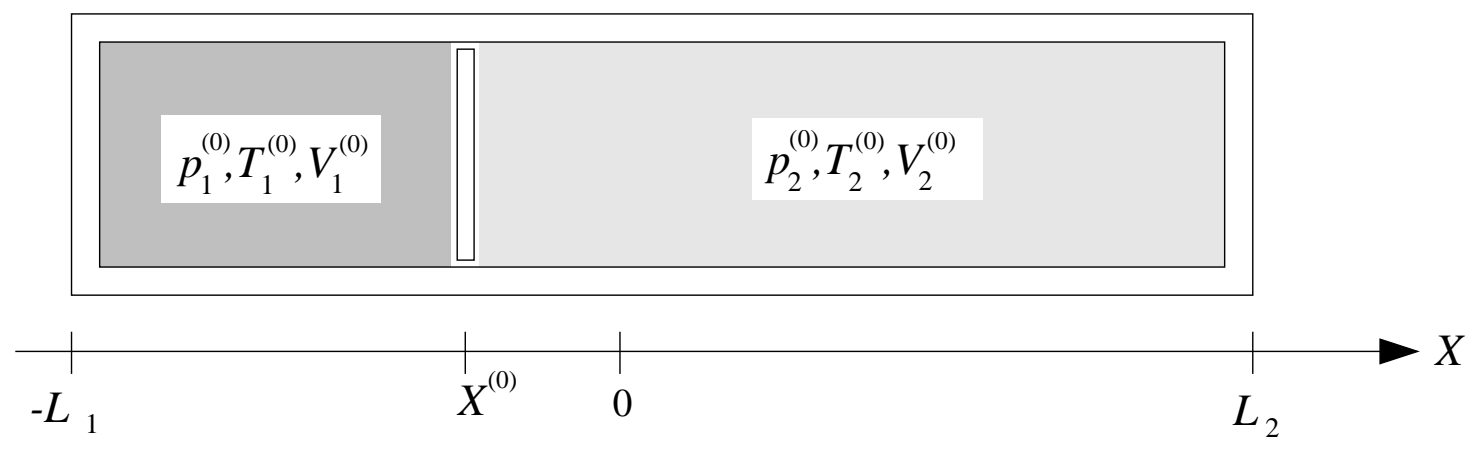

Figure 1: Adiabatic piston problem

The laws of thermostatics give immediately the condition for the mechanical equilibrium, namely that in the final equilibrium state the pressures must be equal, i.e. $p_{1}^{f}=p_{2}^{f}$ (with $\left.E_{1}^{f}+E_{2}^{f}=E_{1}^{0}+E_{2}^{0}\right)$. However, they give no information concerning the final temperatures, except for the condition that they must be such that for both fluids the final entropy cannot be smaller than its initial value, i.e. $S_{1}^{f} \geq S_{1}^{0}$, and $S_{2}^{f} \geq S_{2}^{0}$.

On the other hand, from the laws of thermodynamics one can derive ordinary differential equations for the time evolution of the system. Solving these equations would then give the unique solution of the problem, and in particular the final temperatures. This idea has been recently followed in [3], where a system of thermodynamic evolution equations has been derived within a very simple model. One had to distinguish then between the case 
where the two fluids have vanishing viscosities and the case where at least one of the fluids has a non-zero viscosity. For non-viscous fluids the thermodynamic equations predicts a periodic motion subject to the energy conservation law, with constant subsystem entropies: $S_{1}(t)=S_{1}^{(0)}, S_{2}(t)=S_{2}^{(0)}$. When at least one of the fluids is viscous, the system will attain the final equilibrium state with equal pressures $p_{1}=p_{2}$. However, to find the final entropies (or temperatures) one has to solve the evolution equations which so far could not be achieved explicitly. These same evolution equations were also obtained in the framework of the kinetic theory of gases, assuming the fluctuations of the piston to be negligibly small and considering perfect gases, and several numerical solutions have been obtained 四.

But even having obtained the macroscopic description of the evolution of the system the controversial question remains whether the stochastic frictionless motion of the piston induced by collisions with the fluid particles could eventually lead (on a much longer time scale) to a final state where the temperatures of the fluids are equal, and whether such a microscopic fluctuation mechanism would not violate the second law of thermodynamics. Clearly, the analysis of such problem requires the microscopic description of the dynamical evolution.

In this article we thus present a microscopic study of the motion of the adiabatic piston in contact with two infinite volume reservoirs. Our analysis, based on the Boltzmann equation, has been inspired by the above fundamental adiabatic piston question.

In Section 2 we define the microscopic model and we discuss the applicability of the Boltzmann equation to the description of the motion of the piston. The rigorous determination of the stationary state in one dimension for the case where the mass of the piston is equal to the fluid particle mass is presented in Section 3. The derived solution at equal temperatures reduces simply to the Maxwell equilibrium state. But when the temperatures are different a non-equilibrium stationary state appears characterized by an average velocity of the piston oriented toward the higher temperature region, although no macroscopic force is present (the pressures on both sides of the piston are supposed to be equal). This situation is thus precisely that of the "molecular motor problem". The rigorous derivation (within 
Boltzmann's theory) of the fluctuation induced macroscopic motion is the main result of our work. The relevance of this prediction to a much more realistic problem of a massive piston is discussed in the closing Section 4. The case where the mass of the piston is much larger than that of a fluid particle will be discussed in the forthcoming paper [5].

\section{ADIABATIC PISTON SEPARATING TWO THERMOSTATS: THE BOLTZMANN EQUATION}

In order to simplify the analysis as much as possible we consider in the following the one-dimensional system occupying the whole infinite line. The two fluids are assumed to be perfect gases composed of identical point particles of mass $m$. They are separated by a structureless particle of mass $M$ which plays the role of the piston. The perfectly elastic collisions between the fluid particles result in instantaneous exchanges of velocities. So, from the point of view of the dynamics of the piston the fluid particles can be considered as noninteracting, penetrating each other in their unperturbed free motion. The only perturbation

of the fluid states is due to collisions with the piston. The similar model has been already investigated almost forty years ago by Lebowitz [6] and few years later by Alkemade, van Kampen and Mac Donald [10].

When a collision occurs, the pre-collisional velocities $V$ and $v$ of the piston and the fluid particle, respectively, suffer a change according to the elastic collision law

$$
\begin{gathered}
V \rightarrow V^{\prime}=V+\frac{2 m}{M+m}(v-V) \\
v \rightarrow v^{\prime}=v-\frac{2 M}{M+m}(v-V)
\end{gathered}
$$

Let us suppose that at the initial moment the fluids to the left and to the right of the piston are at equilibrium with temperatures $T_{1}$ and $T_{2}$, and number densities $n_{1}$ and $n_{2}$, respectively. Moreover, the macroscopic mechanical equilibrium will be assumed: the pressures on both sides of the piston are equal

$$
p_{1}=n_{1} k_{B} T_{1}=p_{2}=n_{2} k_{B} T_{2}
$$


( $k_{B}$ is Boltzmann's constant).

When $M \gg m$, the probability that the piston interacts back with the fluid particle it already collided with becomes negligible. In the absence of recollisions the piston always "sees" the unperturbed Maxwell distributions of the arriving fluid particles, and no correlations can occur between its velocity and the precollisional velocity of the fluid particles. The perturbations caused by collisions with the piston go away to infinity. So, in the large mass limit $m / M \rightarrow 0$, we can expect the linear Boltzmann equation to give a correct description of the dynamical evolution of the piston. In order to write down this equation we denote by $\phi_{T_{1}}^{m}$ and $\phi_{T_{2}}^{m}$ the Maxwell velocity distributions of the two surrounding fluids, where

$$
\phi_{T}^{m}=\sqrt{\frac{m}{2 \pi k_{B} T}} \exp \left(-\frac{m v^{2}}{2 k_{B} T}\right)
$$

Let $F(X, V ; t)$ denote the probability density for finding the piston at point $X$ with velocity $V$ at time $t$. When $m / M \ll 1, F(X, V ; t)$ satisfies the linear Boltzmann equation of the form

$$
\begin{aligned}
& \left(\frac{\partial}{\partial t}+V \frac{\partial}{\partial X}\right) F(X, V ; t)=\int d v|V-v| \times \\
& {\left[\theta(V-v) n_{1} \phi_{T_{1}}^{m}\left(v-\frac{2 M}{M+m}(v-V)\right) F\left(X, V+\frac{2 m}{M+m}(v-V) ; t\right)\right.} \\
& -\theta(v-V) n_{1} \phi_{T_{1}}^{m}(v) F(X, V ; t) \\
& +\theta(v-V) n_{2} \phi_{T_{2}}^{m}\left(v-\frac{2 M}{M+m}(v-V)\right) F\left(X, V+\frac{2 m}{M+m}(v-V) ; t\right) \\
& \left.-\theta(V-v) n_{2} \phi_{T_{2}}^{m}(v) F(X, V ; t)\right]
\end{aligned}
$$

where $\theta$ is the unit Heaviside step function.

Our aim is to determine the velocity distribution of the piston in the asymptotic stationary state attained for $t \rightarrow \infty$. It turns out that this problem can be given a rigorous answer in the special case of $M=m$. But when the mass $M$ of the piston is equal to the fluid particle mass $m$, the recollisions can take place, and the validity of the Boltzmann equation becomes questionable. However, what we are really interested in is the case of $M \gg m$. We thus expect that neglecting the effect of recollisions in the equal mass case will lead to the 
evolution qualitatively similar to that occuring for $M \gg m$, simply because the same class of dynamical events will be taken into account. This should apply in particular to the nature of the stationary state. It should be noticed here that even when compared with the exact results the linear Boltzmann equation for $M=m$ represented a very reasonable approximation to the velocity correlation function and to the Van Hove self-correlation function of the one-dimensional hard rod fluid [7].

Having adopted the above point of view we are going to study in the next section the equation satisfied by the stationary probability density $\Phi(V)$ for finding the piston with velocity $V$ in the equal pressure case (see eq.(2)). Denoting by $F(X, V)$ the stationary solution of equation (雨), we have

$$
\Phi(V)=\int d X F(X, V)
$$

\section{SOLVING THE BOLTZMANN EQUATION: APPEARANCE OF A MOLECULAR MOTOR}

According to the linear Boltzmann equation (4) the stationary velocity distribution $\Phi(V)$ in the equal pressure case (2) satisfies the equation

$$
\begin{aligned}
& \int d v|V-v|\left[\theta(v-V) \frac{1}{T_{1}} \phi_{T_{1}}^{m}(v)+\theta(V-v) \frac{1}{T_{2}} \phi_{T_{2}}^{m}(v)\right] \Phi(V) \\
& =\int d v|V-v|\left[\theta(V-v) \frac{1}{T_{1}} \phi_{T_{1}}^{m}(V)+\theta(v-V) \frac{1}{T_{2}} \phi_{T_{2}}^{m}(V)\right] \Phi(v)
\end{aligned}
$$

In the following we shall assume $T_{2}>T_{1}$.

It is convenient to express eq.(6) in terms of the following two functions

$$
\begin{aligned}
I(V) & =\int_{V}^{\infty} d v(v-V) \frac{1}{T_{1}} \phi_{T_{1}}^{m}(v)+\int_{-\infty}^{V} d v(V-v) \frac{1}{T_{2}} \phi_{T_{2}}^{m}(v) \\
G(V) & =\int_{V}^{\infty} d v(v-V) \Phi(v)+\int_{-\infty}^{V} d v(V-v) \Phi(v)
\end{aligned}
$$

Using the identities 


$$
\begin{aligned}
& |V-v| \theta(V-v)=\frac{1}{2}[(V-v)+|V-v|] \\
& |V-v| \theta(v-V)=\frac{1}{2}[-(V-v)+|V-v|]
\end{aligned}
$$

we rewrite eq.(6) in the form

$$
I(V) \Phi(V)-\frac{1}{2}\left[\frac{1}{T_{1}} \phi_{T_{1}}^{m}(V)+\frac{1}{T_{2}} \phi_{T_{2}}^{m}(V)\right] G(V)=\frac{1}{2}(V-<V>)\left[\frac{1}{T_{1}} \phi_{T_{1}}^{m}(V)-\frac{1}{T_{2}} \phi_{T_{2}}^{m}(V)\right]
$$

where $\langle V\rangle$ denotes the average velocity of the piston

$$
<V>=\int d V \Phi(V) V
$$

We now notice that the first- and second-order derivatives of the functions $I(V)$ and $G(V)$ are given by

$$
\begin{aligned}
I^{\prime}(V) & =-\int_{V}^{\infty} d v \frac{1}{T_{1}} \phi_{T_{1}}^{m}(v)+\int_{-\infty}^{V} d v \frac{1}{T_{2}} \phi_{T_{2}}^{m}(v) \\
I^{\prime \prime}(V) & =\frac{1}{T_{1}} \phi_{T_{1}}^{m}(v)+\frac{1}{T_{2}} \phi_{T_{2}}^{m}(v) \\
G^{\prime}(V) & =-\int_{V}^{\infty} d v \Phi(v)+\int_{-\infty}^{V} d v \Phi(v) \\
G^{\prime \prime}(V) & =2 \Phi(V)
\end{aligned}
$$

Therefore (9) takes the form of a simple differential equation

$$
\begin{gathered}
I(V) G^{\prime \prime}(V)-I^{\prime \prime}(V) G(V)=\left[I(V) G^{\prime}(V)-I^{\prime}(V) G(V)\right]^{\prime} \\
=(V-<V>)\left[\frac{1}{T_{1}} \phi_{T_{1}}^{m}(V)-\frac{1}{T_{2}} \phi_{T_{2}}^{m}(V)\right]
\end{gathered}
$$

Using the asymptotic formulae

$$
\begin{gathered}
I(V) \rightarrow\left\{\begin{array}{r}
\frac{1}{T_{2}} V, \text { for } V \rightarrow+\infty \\
-\frac{1}{T_{1}} V, \text { for } V \rightarrow-\infty
\end{array}\right. \\
G(V) \rightarrow\left\{\begin{array}{r}
(V-<V>), \text { for } V \rightarrow+\infty \\
-(V-<V>), \text { for } V \rightarrow-\infty
\end{array}\right.
\end{gathered}
$$


we can readily evaluate the limit

$$
\lim _{V \rightarrow \pm \infty}\left[I(V) G^{\prime}(V)-I^{\prime}(V) G(V)\right]=\left(\frac{1}{T_{1}}+\frac{1}{T_{2}}\right)<V>
$$

Integrating equation (15) with the boundary condition (18) yields the relation

$$
\begin{aligned}
I(V) G^{\prime}(V)-I^{\prime}(V) G(V) & =\frac{k_{B}}{m}\left[\phi_{T_{2}}^{m}(V)-\phi_{T_{1}}^{m}(V)\right] \\
& +<V>\left[\int_{V}^{\infty} d v \frac{1}{T_{1}} \phi_{T_{1}}^{m}(v)+\int_{-\infty}^{V} d v \frac{1}{T_{2}} \phi_{T_{2}}^{m}(v)\right]
\end{aligned}
$$

We thus arrived at a first order differential equation for the unknown function $G$. The solution of eq.(19) can be now constructed by standard methods. Introducing the auxiliary function $H(V)$

$$
G(V)=I(V) H(V)
$$

and using the relations

$$
\begin{aligned}
& \lim _{V \rightarrow+\infty} H(V)=\lim _{V \rightarrow+\infty} \frac{G(V)}{I(V)}=T_{2} \\
& \lim _{V \rightarrow-\infty} H(V)=\lim _{V \rightarrow-\infty} \frac{G(V)}{I(V)}=T_{1}
\end{aligned}
$$

one readily determines the function $H$. It reads

$$
\begin{aligned}
2 H(V) & =T_{1}+T_{2}+\int \frac{d u}{I^{2}(u)} \operatorname{sgn}(V-u) \frac{k_{B}}{m}\left[\phi_{T_{2}}^{m}(u)-\phi_{T_{1}}^{m}(u)\right] \\
& +\int \frac{d u}{I^{2}(u)} \operatorname{sgn}(V-u)\left[\int_{-\infty}^{u} d v \frac{1}{T_{2}} \phi_{T_{2}}^{m}(v)+\int_{u}^{\infty} d v \frac{1}{T_{1}} \phi_{T_{1}}^{m}(v)\right]<V>
\end{aligned}
$$

Here $\operatorname{sgn}(V-u)=\theta(V-u)-\theta(u-V)$. The relations (14), (20) permit to determine the shape of the stationary probability density $\Phi(V)$. We find

$$
\begin{gathered}
\Phi(V)=\frac{(V-<V>)}{2 I(V)}\left[\frac{1}{T_{1}} \phi_{T_{1}}^{m}(V)-\frac{1}{T_{2}} \phi_{T_{2}}^{m}(V)\right] \\
+\frac{1}{4}\left[\frac{1}{T_{1}} \phi_{T_{1}}^{m}(V)+\frac{1}{T_{2}} \phi_{T_{2}}^{m}(V)\right]\left\{T_{1}+T_{2}+\right. \\
\left.+\int \frac{d u}{I^{2}(u)} \operatorname{sgn}(V-u)\left[\frac{k_{B}}{m}\left(\phi_{T_{2}}^{m}(u)-\phi_{T_{1}}^{m}(u)\right)+<V>\left(\int_{-\infty}^{u} d v \frac{1}{T_{2}} \phi_{T_{2}}^{m}(v)+\int_{u}^{\infty} d v \frac{1}{T_{1}} \phi_{T_{1}}^{m}(v)\right)\right]\right\}
\end{gathered}
$$


In order to complete the calculation of $\Phi(V)$ we have still to evaluate the average piston velocity $\langle V\rangle$ of the piston which shows in equation (23). This can be achieved from the normalization condition

$$
\int d V \Phi(V)=1
$$

To perform the calculation we group together the terms multiplying $\langle V\rangle$, and rewrite equation (23) in the form

$$
K(V)<V>=L(V)
$$

It turns out that the function $K(V)$ can be written as a derivative

$$
\begin{aligned}
K(V) & =\frac{\partial}{\partial V}\left[\frac{1}{I(V)}\left(\int_{-\infty}^{V} d v \frac{1}{T_{2}} \phi_{T_{2}}^{m}(v)+\int_{V}^{\infty} d v \frac{1}{T_{1}} \phi_{T_{1}}^{m}(v)\right)\right. \\
& \left.+\frac{1}{2} I^{\prime}(V) \int \frac{d u}{I^{2}(u)} \operatorname{sgn}(V-u)\left(\int_{-\infty}^{u} d v \frac{1}{T_{2}} \phi_{T_{2}}^{m}(v)+\int_{u}^{\infty} \frac{1}{T_{1}} \phi_{T_{1}}^{m}(v)\right)\right]
\end{aligned}
$$

We then deduce from $(26)$ the relation

$$
\int_{-\infty}^{+\infty} d V K(V)=\frac{1}{2}\left(\frac{1}{T_{2}}-\frac{1}{T_{1}}\right) \int_{-\infty}^{+\infty} \frac{d u}{I^{2}(u)}\left(\int_{-\infty}^{u} d v \frac{1}{T_{2}} \phi_{T_{2}}^{m}(v)+\int_{u}^{\infty} d v \frac{1}{T_{1}} \phi_{T_{1}}^{m}(v)\right)
$$

which follows directly from the asymptotic formulae (16). The right hand side of equation (25) equals

$$
L(V)=2 \Phi(V)-\frac{1}{2}\left(T_{1}+T_{2}\right)\left[\frac{1}{T_{1}} \phi_{T_{1}}^{m}(V)+\frac{1}{T_{2}} \phi_{T_{2}}^{m}(V)\right]-\frac{k_{B}}{m} \frac{\partial}{\partial V} A(V)
$$

where

$$
\begin{gathered}
A(V)=\frac{1}{I(V)}\left[\phi_{T_{2}}^{m}(V)-\phi_{T_{1}}^{m}(V)\right]+ \\
+\frac{1}{2}\left(\int_{-\infty}^{V} d v \frac{1}{T_{2}} \phi_{T_{2}}^{m}(v)-\int_{V}^{\infty} d v \frac{1}{T_{1}} \phi_{T_{1}}^{m}(v)\right) \int \frac{d v}{I^{2}(v)} \operatorname{sgn}(V-v)\left[\phi_{T_{2}}^{m}(v)-\phi_{T_{1}}^{m}(v)\right]
\end{gathered}
$$

Taking again into account the asymptotics (16) and the normalization (24) we get

$$
\int_{-\infty}^{+\infty} d V L(V)=-\frac{\left(T_{1}-T_{2}\right)}{2 T_{1} T_{2}}\left[T_{1}-T_{2}+\frac{k_{B}}{m} \int_{-\infty}^{+\infty} \frac{d v}{I^{2}(v)}\left(\phi_{T_{2}}^{m}(v)-\phi_{T_{1}}^{m}(v)\right)\right]
$$


Integrating equation (25) over the whole velocity space we thus arrive at the following formula for the stationary mean velocity of the piston

$$
\begin{aligned}
<V> & =\left\{T_{2}-T_{1}+\frac{k_{B}}{m} \int_{-\infty}^{+\infty} \frac{d v}{I^{2}(v)}\left[\phi_{T_{1}}^{m}(v)-\phi_{T_{2}}^{m}(v)\right)\right\} \\
& \times\left\{\int_{-\infty}^{+\infty} \frac{d u}{I^{2}(u)}\left[\int_{-\infty}^{u} d v \frac{1}{T_{2}} \phi_{T_{2}}^{m}(v)+\int_{u}^{\infty} d v \frac{1}{T_{1}} \phi_{T_{1}}^{m}(v)\right]\right\}^{-1}
\end{aligned}
$$

The formula (30) is the main result of our analysis. Whereas the mean velocity vanishes for $T_{1}=T_{2}$, the piston acquires a macroscopic systematic motion when $T_{1} \neq T_{2}$, and this under the equal pressures condition (2).

In order to further investigate this phenomenon let us analyze the case of a small temperature difference. We thus put

$$
T_{1}=T, \quad T_{2}=T+\delta T, \text { with } 0<\frac{\delta T}{T} \ll 1
$$

When $\delta T \rightarrow 0$, the denominator in equation (30) takes the asymptotic form

$$
T \sqrt{\frac{m}{k_{B} T}} \int d w h(w)+\mathrm{O}(\delta T)
$$

where

$$
h(w)=\left[\int d w|u-w| \phi(w)\right]^{-2}
$$

and $\phi(w)$ is the dimensionless Maxwell distribution

$$
\phi(w)=\frac{1}{\sqrt{2 \pi}} \exp \left(-w^{2} / 2\right)
$$

On the other hand the numerator equals

$$
\delta T\left[1-\frac{1}{2} \int d w h(w)\left(w^{2}-1\right) \phi(w)\right]+\mathrm{O}\left((\delta T)^{2}\right)
$$

So, when $\delta T \rightarrow 0$, the mean velocity of the piston is given by

$$
<V>=\sqrt{\frac{k_{B} T}{m}}\left(\frac{\delta T}{T}\right)\left[1-\frac{1}{2} \int d w h(w)\left(w^{2}-1\right) \phi(w)\right]\left[\int d w h(w)\right]^{-1}+\mathrm{O}(\delta T)
$$

Integration by parts yields the relation 


$$
\int d w h(w)\left(w^{2}-1\right) \phi(w)=\int d w w \phi(w) h^{\prime}(w)
$$

The inequality

$$
w h^{\prime}(w)=-2[h(w)]^{3 / 2} w \int d w^{\prime} \operatorname{sign}\left(w-w^{\prime}\right) \phi\left(w^{\prime}\right) \leq 0
$$

permits to determine the sign of the mean velocity of the piston. We find

$$
<V>>0, \quad \text { if } \delta T>0
$$

The inequality (36) shows that in the stationary state the piston performs an uniform motion in the direction of the higher temperature region. In fact, we checked by numerical analysis that the inequality (36) remained true for any $T_{2}>T_{1}$.

\section{CONCLUDING COMMENTS}

We have studied the effect of fluctuations on the motion of the piston separating two semi-infinite regions filled with perfect gases at equal pressures. The explicit stationary solution of the Boltzmann equation (23), (30) obtained for the special case where the mass of the piston equals that of a fluid particle reduces to the equilibrium Maxwell distribution when the temperatures on both sides of the piston are equal. The mean velocity then vanishes. In fact, it has been rigorously shown that the piston (tagged particle) performs in this case a clasical diffusive motion [8], [9].

When the temperatures are different, the space asymmetry induces asymmetry of fluctuations which turns out to have a macroscopic effect. The piston attains a stationary state with a non-zero average velocity oriented toward the higher temperature region. In view of the absence of macroscopic forces we discover here a genuine molecular motor. There is some analogy between our prediction and the "stochastic ratchet" problem where one observes that fluctuations conspire with the spatial anisotropy to induce a macroscopic directed motion in the absence of macroscopic forces [11], [12]. 
We expect the same qualitative behavior in the case of a massive piston whose mass is much larger than that of a gas molecule. Indeed, when $M \gg m$ the Boltzmann equation, which was the basis of our analysis, gives an adequate description of the dynamics because the creation of dynamical correlations between the piston and the gas molecules via the recollision mechanism becomes negligible. The persistence of the non-vanishing average velocity in the $M \gg m$ regime at equal pressures and different temperatures will be the subject of the forthcoming article [5]. One can thus expect that the stochastic motion of the piston as a whole can lead to an effective energy transfer between the separated fluids in the absence of pressure difference. Hence, although the piston has no internal degrees of freedom calling it adiabatic becomes a misuse of the term.

It remains to be seen what is the relevance of our analysis to the original adiabatic piston problem where the fluids occupy finite volumes. This is a delicate question indeed, because in one dimension even for a very massive piston the collisions with the boundaries will inevitably induce recollisions: the piston will interact back with the particles it had already met before. So, the Boltzmann description cannot be reliable in the long time limit. Indeed, at the time scale characterized by the time necessary to cover the total length of the cylinder $\left(L_{1}+L_{2}\right)$ with the thermal velocity of the fluids the effects of dynamical correlations will start to play an important role. However, for sufficiently large volumes, after the mechanical equilibrium has been established, one can expect (on a larger time scale) the occurence of a net displacement of the piston, reducing the volume of the the higher temperature fluid, as it is the case for the conducting piston. It seems likely that for finite volumes the Boltzmann description can work much better in two and three dimensions because then the recollision effects due to boundaries become weaker. Anyway, for finite volumes one will have to consider the system of kinetic equations coupling the evolution of the state of the piston to that of the fluids. 


\section{REFERENCES}

1 The authors are greatful to J.L Lebowitz and N.G. van Kampen for stimulating information about their own work on a similar model.

2 J.Piasecki greatly acknowledges the hospitality at the Institue of Theoretical Physics of the École Polytechnique Fédérale de Lausanne where a part of this research has been performed.

[1] E. Lieb and J. Yngvason, The physics and mathematics of the second law of thermodynamics, Physics Reports (in press), Austin, Math.Phys. Archive.

[2] H.B. Callen, Thermodynamics, John Wiley and Sons, New York, 1963. Appendix C. See also H.B. Callen, Thermodynamics and Thermostatics, John Wiley and Sons, 2nd edition, New York, 1985, pp. 51 and 53.

[3] Ch. Gruber, Thermodynamics of systems with internal adiabatic constrains: time evolution of the adiabatic piston, preprint EPFL (1998).

[4] B. Crosignani, P. Di Porto and M. Segev, Approach to thermal equilibrium in a system with adiabatic constraint, Am.J.Phys. 64 (1996), 610.

[5] Ch.Gruber and J.Piasecki (in preparation).

[6] J.L. Lebowitz, Stationary non-equilibrium Gibbsian ensembles, Phys.Rev. 114 (1959) 1192.

[7] P. Résibois, Solution de l'équation de Boltzmann pour des bâtonnets durs unidimensionnels, Physica 90A (1978) 273.

[8] D.W. Jepsen, Dynamics of a simple many-body system of hard rods, J.Math.Phys. 6 (1965) 405 .

[9] J.L. Lebowitz and J.K. Percus, Kinetic equations and density expansions: exactly solvable one-dimensional system, Phys.Rev. 155 (1967) 122. 
[10] C.T.J. Alkemade, N.G. van Kampen and D.K.C. Mac Donald, F.R.S., Non-linear Brownian movement of a generalized Rayleigh model, Proc.Roy.Soc. A271 (1963) 449.

[11] E. Abad and A. Mielke, Brownian motion in fluctuating periodic potentials, Ann.Physik 7 (1998) 9 .

[12] H. Kohler and A. Mielke, Noise-induced transport at zero temperature, J.Phys.A 31 (1998) 1929. 